\title{
Pion-pion scattering lengths determination from kaon decays
}

\author{
Sergey Gevorkyan \\ Joint Institute for Nuclear Research, Dubna, Russia and Yerevan Physics Institute,Armenia \\ E-mail: gevs@jinr.ru
}

Final state interactions of pions in the decay $K^{ \pm} \rightarrow \pi^{ \pm} \pi^{0} \pi^{0}$ lead to the anomaly (cusp) in the $\pi^{0} \pi^{0}$ invariant mass distribution in the vicinity of charged pions threshold. We present our results of accounting of the electromagnetic interaction among charged pions leading to bound states (pionium atom) just under the charged pions threshold .

The isospin breaking effects in the decay $K^{ \pm} \rightarrow \pi^{+} \pi^{-} e^{ \pm} v$ ( $K_{e 4}$ decay) are shortly considered.

The 7th International Workshop on Chiral Dynamics,

August 6 -10, 2012

Jefferson Lab, Newport News, Virginia, USA 


\section{Scattering lengths as a test of chiral symmetry breaking}

The pion-pion scattering at low energies provides a testing ground for strong interaction study. As the free pion targets cannot be created the experimental evaluation of $\pi \pi$ scattering characteristics is restricted to the study of a dipion system in a final state of more complicated reactions. One of the best way for $\pi \pi$ scattering lengths determination is the kaon decays to two or three pions in the final state. The Chiral Perturbation Theory (ChPT), which is a systematic expansion of Green functions in terms of external momenta and quark masses claims to be the theory of strong interaction at low energies. At present it predicts the values of pion-pion s-wave scattering lengths $a_{0}, a_{2}(\pi \pi$ state isospin $\mathrm{I}=0,2)$ with unusual for strong interaction precision [1]. For instance ${ }^{1}$ $a_{0}=0.22 \pm 0.005$. To check these predictions one has to extract the scattering lengths values from experimental data with comparable accuracy. The importance of precise knowledge of scattering lengths is due to the fact that their values give information on the degree of chiral symmetry breaking. In the world with exact chiral symmetry $\left(m_{u}=m_{d}=0\right)$ the scattering lengths should be zero. Moreover their values allows one to estimate the size of the quark-antiquark condensate. For instance in the standard ChPT with large condensate the value of isoscalar s-wave scattering length should be about $a_{0}=0.2$, whereas in generalized ChPT with small condensate its value should be around 0.3 .

\section{Cusp in $K^{ \pm} \rightarrow \pi^{ \pm} \pi^{0} \pi^{0}$ decay}

In 2003-2004 the collaboration NA48/2 at CERN SPS collected a large amount of data [2] on the decay $K^{ \pm} \rightarrow \pi^{ \pm} \pi^{0} \pi^{0}$. The dependence of its distribution on the invariant mass of the two neutral pions $M_{00}$ (fig. 1) shows a cusp-like anomaly in the vicinity of charged pions threshold $M_{00}=2 m_{c}$. As was pointed out by N.Cabibbo [3] this anomaly is a result of charge exchange

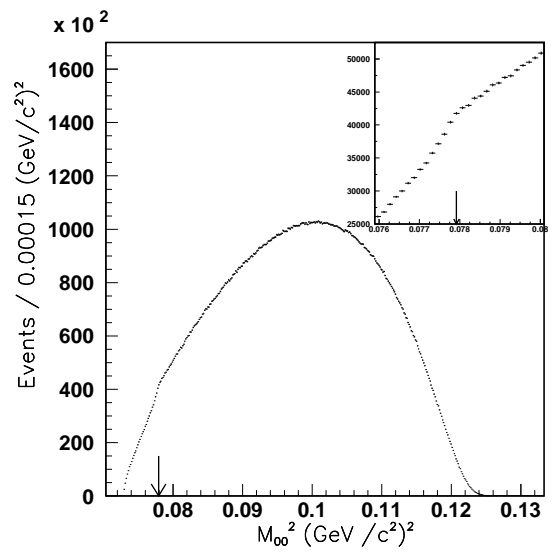

Figure 1: Distribution of $M_{00}^{2}$ the square of the $\pi^{0} \pi^{0}$ invariant mass.

process $\pi^{+} \pi^{-} \rightarrow \pi^{0} \pi^{0}$ in the decay $K^{ \pm} \rightarrow \pi^{ \pm} \pi^{+} \pi^{-}$. The amplitude of the decay $K^{+} \rightarrow \pi^{+} \pi^{0} \pi^{0}$

\footnotetext{
${ }^{1}$ In units of inverse charged pion mass $m_{c}^{-1}$.
} 
reads:

$$
T=T_{0}+2 i a_{x} k_{c} T_{+}, \quad a_{x}\left(\pi^{+} \pi^{-} \rightarrow \pi^{0} \pi^{0}\right)=\frac{a_{2}-a_{0}}{3},
$$

$T_{0}\left(K^{+} \rightarrow \pi^{+} \pi^{0} \pi^{0}\right), T_{+}\left(K^{+} \rightarrow \pi^{+} \pi^{-} \pi^{+}\right)$are unperturbed (without final state interaction) amplitudes, $k_{c}=\frac{1}{2} \sqrt{M_{00}^{2}-4 m_{c}^{2}}$ is the relative momentum of charged pion in the reaction $\pi^{+}+\pi^{-} \rightarrow$ $\pi^{0}+\pi^{0}$.

Under charged pions threshold this momentum becomes imaginary $k_{c}=i \kappa$ :

$$
\begin{aligned}
& |T|^{2}=T_{0}^{2}+4 \frac{\left(a_{0}-a_{2}\right)^{2} k^{2}}{9} T_{+}^{2}, \quad M_{00}^{2}>4 m_{c}^{2}, \\
& |T|^{2}=T_{0}^{2}+4 \frac{\left(a_{0}-a_{2}\right)^{2} \kappa^{2}}{9} T_{+}^{2}-4 \frac{\left(a_{0}-a_{2}\right) \kappa}{3} T_{0} T_{+}, \quad M_{00}^{2}<4 m_{c}^{2} .
\end{aligned}
$$

Thus above the charged pions threshold $M_{00}^{2}>4 m_{c}^{2}$ the decay rate is proportional to the square of scattering lengths difference $\left(a_{0}-a_{2}\right)^{2}$, the fact known for many years [4], while under threshold $M_{00}^{2}<4 m_{c}^{2}$ the decay rate acquires interference term linear in $\left(a_{0}-a_{2}\right)$. The presence of cusp in the decay $K^{ \pm} \rightarrow \pi^{ \pm} \pi^{0} \pi^{0}$ allows one to extract the value of s-wave $\pi \pi$ scattering lengths difference $a_{0}-a_{2}$ with high precision.

For many years the semileptonic decay $K^{ \pm} \rightarrow \pi^{+} \pi^{-} e v$ ( $K_{e 4}$ decay) was seen as a cleanest method for measurement of $\pi \pi$ scattering lengths, due to only two pions in the final state and well known connection between the pions phases difference in $\mathrm{s}$ and $\mathrm{p}$ wave states with scattering lengths. The discovery of cusp effect by NA48/2 collaboration open a new challenges for precise determination of scattering lengths.

The fit of the experimental distribution making use of two theoretical models $[5,6]$ present the extension of N.Cabibbo's approach to higher order in scattering lengths $\left(\sim a^{2}\right)$ allows to determine the scattering lengths with accuracy comparable to theoretical predictions.

Nevertheless there are two problems that have been unsolved in the above consideration. At charge pions threshold one has to account for electromagnetic interaction of pions leading to bound states (pionium atoms) just under threshold. In the fig.2a the result of the fit without electromagnetic effects is shown [2]. The discrepancy in the vicinity of charged pions threshold is a result of neglect of this effect in the theoretical approaches. The better fit turn out ( fig.2b) if one adds a free parameter relevant to a term describing the expected formation of pionium atoms decaying to $\pi^{0} \pi^{0}$ at threshold. However the obtained in such way value of $K^{ \pm} \rightarrow \pi^{ \pm}+\left(\pi^{+} \pi^{-}\right)$normalized to the $K^{ \pm} \rightarrow \pi^{ \pm} \pi^{+} \pi^{-}$decay rate turn out to be twice larger than theoretical prediction.

\section{Higher order effects and electromagnetic interactions}

The second issue closely connected to bound state formation is the absence of a reliable way to estimate the contribution of higher order terms in strong interaction. The reason is that to account for electromagnetic interaction leading to unstable bound states one needs expressions for decay amplitudes including the strong interaction between pions in all orders, the task which can't be solved in the framework of two mentioned above $[5,6]$ theoretical models. 


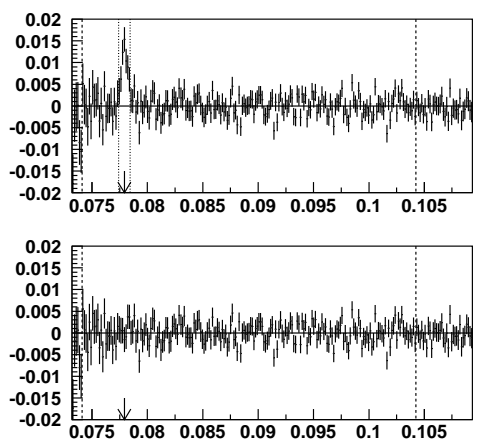

Figure 2: $\Delta=\left(\right.$ data-fit)/data versus $M_{00}^{2}$ : a-fit without pionium formation; b-fit including the electromagnetic effects.

To resolve these problems, we make use of the well known methods of non-relativistic quantum mechanics, obtaining [7] the general expressions for the decay amplitudes to the two different channels $\left(\pi^{+} \pi^{-}\right.$and $\left.\pi^{0} \pi^{0}\right)$ accounting for the strong interaction between pions to all orders. The general form of matrix element for kaon decay to the final state with two charged or neutral pions can be written in the operator form:

$$
M_{c}=\int \Psi_{c}^{+}(r) M_{0}(r) d^{3} r ; \quad M_{n}=\int \Psi_{n}^{+}(r) M_{0}(r) d^{3} r
$$

The two component operator $M_{0}=\left(\begin{array}{c}M_{c}^{(0)}(r) \\ M_{n}^{(0)}(r)\end{array}\right)$, where $M_{c}^{(0)}(r), M_{n}^{(0)}(r)$ are the matrix elements of kaon decay to noninteracting charged and neutral pions pairs, while $\Psi_{c}(r), \Psi_{n}(r)$ are the appropriate two component wave functions.

These wave functions would satisfy to couple Shrôdinger equations ${ }^{2}$

$$
\begin{aligned}
& -\Delta \Psi_{c}(r)+U_{c c} \Psi_{c}(r)+U_{c n} \Psi_{n}(r)=k_{c}^{2} \Psi_{c}(r) \\
& -\Delta \Psi_{n}(r)+U_{n n} \Psi_{n}(r)+U_{n c} \Psi_{c}(r)=k_{n}^{2} \Psi_{n}(r)
\end{aligned}
$$

where $U_{i j}$ are the strong potentials describing elastic $c c \rightarrow c c ; n n \rightarrow n n$ scattering and charge exchange reaction $c n \rightarrow c n . k_{c}, k_{n}$ are the charge and neutral pions momenta in the appropriate center of mass system.

Assuming the strong potentials with sharp boundary $U_{i k} \gg k_{c(n)}^{2}$, making use the known asymptotic behavior of wave functions and unitarity constrains we obtain a set of relations expressing the decay amplitudes through unperturbed one $M_{0 c}, M_{0 n}$ and $\pi \pi$ scattering amplitudes $f_{x}\left(\pi^{+} \pi^{-} \rightarrow\right.$ $\left.\pi^{0} \pi^{0}\right), \quad f_{c}\left(\pi^{+} \pi^{-} \rightarrow \pi^{+} \pi^{-}\right), \quad f_{n}\left(\pi^{0} \pi^{0} \rightarrow \pi^{0} \pi^{0}\right)$

$$
M_{c}=M_{0 c}\left(1+i k_{c} f_{c c}\right)+i k_{n} M_{0 n} f_{x}, \quad f_{c c}=\frac{a_{c c}\left(1-i k_{n} a_{n n}\right)+i k_{n} a_{x}^{2}}{D},
$$

\footnotetext{
${ }^{2}$ We restricted by s-wave $\pi \pi$ scattering in the final state.
} 


$$
\begin{aligned}
M_{n} & =M_{0 n}\left(1+i k_{n} f_{n n}\right)+i k_{c} M_{0 c} f_{x}, \quad f_{n n}=\frac{a_{n n}\left(1-i k_{c} a_{c c}\right)+i k_{c} a_{x}^{2}}{D} \\
f_{x} & =\frac{a_{x}}{D}, \quad D=\left(1-i k_{c} a_{c c}\right)\left(1-i k_{n} a_{n n}\right)+k_{n} k_{c} a_{x}^{2}
\end{aligned}
$$

In order to take into account the electromagnetic interactions between pions, we take an advantage of the trick successfully used in $[8,9]$. To switch on the electromagnetic interaction [10] it is enough to replace the charged pion momenta $k_{c}$ in above expressions by a logarithmic derivative of the pion wave function in the Coulomb potential at the boundary of the strong field $r_{0}$ :

$$
i k_{c} \rightarrow \tau=\left.\frac{d \log \left[G_{0}(k r)+i F_{0}(k r)\right]}{d r}\right|_{r=r_{0}} .
$$

Here $F_{0}, G_{0}$ are the regular and irregular solutions of the Coulomb problem.

In the region $k r_{0} \ll 1$, where the electromagnetic effects are significant, this expression can be simplified:

$$
\begin{aligned}
\tau & =i k-\alpha m\left[\log \left(-2 i k r_{0}\right)+2 \gamma+\psi(1-i \xi)\right] \\
& =\operatorname{Re} \tau+i \operatorname{Im} \tau \\
\operatorname{Re} \tau & =-\alpha m\left[\log \left(2 k r_{0}\right)+2 \gamma+\operatorname{Re} \psi(1-i \xi)\right], \xi=\frac{\alpha m}{2 k} \\
\operatorname{Im} \tau & =k A^{2}, \quad A=\exp \left(\frac{\pi \xi}{2}\right)|\Gamma(1+i \xi)| .
\end{aligned}
$$

Here $\gamma=0.5772$ Euler constant and digamma function $\psi(\xi)=\frac{d \log \Gamma(\xi)}{d \xi}$.

Inclusion of the electromagnetic interactions in thus way lead to the bound state in the very narrow region under charged pions threshold and correctly accounts for the electromagnetic interaction in all kinematical region [10]. It seems that the difference between predicted rate for creation of pionium atoms and fit result at threshold is due to the fact that in the vicinity of threshold besides the bound states, there are contribution from unbound pairs, whose electromagnetic interaction gives almost the same size contribution as the pionium decay [11].

\section{4. $K_{e 4}$ decay}

As was mentioned above the decay $K_{e 4}$ is ideally suited for determination of $\pi \pi$ scattering lengths. The preliminary analysis of the experimental data obtained by NA48/2 collaboration leads to large discrepancies between the predictions for scattering lengths of Chiral Perturbation Theory and their values extracting from experimental data [12].

It was shown [13] that this difference is due to the neglect of isospin breaking effects in the experimental data processing. Account of electromagnetic interaction between the pions in the decay $K^{ \pm} \rightarrow \pi^{+} \pi^{-} e v$ and isospin breaking effects provided by possibility of charge exchange among the pions in the final state allows to adjust the data for scattering lengths from NA48/2 and theoretical predictions. 


\section{Conclusion}

The general principles of non-relativistic quantum mechanics allows one to obtain a compact analytical expressions for decay amplitudes accounting for pion-pion final state interaction to all orders in strong interactions. The developed approach gives a possibility to estimate the contribution of higher order terms in strong interaction of final pions, which is an actual issue accounting for the high precision of current experimental data.

From the other hand the obtained expressions for decay amplitudes allows one to take into account in the correct way the electromagnetic interactions of pions and obtain the formulas describing the bound state (pionium) under the charged pions production threshold. The considered approach can be applied to the wide class of the decays with two or more hadrons in the final state, leading to better understanding of strong interaction and giving the unique possibility to check different theoretical models predictions for meson meson interactions at low energies.

\section{References}

[1] G. Colangelo, J. Gasser, H. Leutwyler, Nucl. Phys. B603, 125 (2001)

[2] J.Batley et al., Phys. Lett. B 633, 173 (2006); Eur. Phys. J. C64, 589(2009)

[3] N. Cabibbo, Phys. Rev. Lett., 93, 121801 (2004)

[4] V. Gribov, Nucl. Phys. 5, 653 (1958)

[5] N. Cabibbo, G. Isidori, JHEP 0503, 021 (2005)

[6] G.Colangelo, J.Gasser, B.Kubis, A.Rusetsky, Phys. Lett. B 638,187 (2006)

[7] S.Gevorkyan, A.Tarasov, O.Voskresenskaya, Eur.Phys. J. C67,143 (2010)

[8] E. Wigner, Phys. Rev. 73, 1002 (1948)

[9] A. Baz, ZhETP 33, 923 (1957)

[10] S.Gevorkyan, A.Tarasov, O.Voskresenskaya, Phys. Lett. B649, 159 (2007)

[11] S.Gevorkyan, D. Madigozhin, A.Tarasov, O.Voskresenskaya, Phys. Part. Nucl. Lett. 5, 85 (2008)

[12] B. Bloch-Devaux, Proceedings of the Kaon International Conference, May, 2007

[13] S.Gevorkyan, A. Sissakian, A. Tarasov, H. Torosyan, O. Voskresenskaya, Phys. Atom. Nucl. 73,961 (2010); 73,937 (2010) 